\title{
Immediate and long term outcome after infrathalamic and thalamic lesioning for intractable Tourette's syndrome
}

\author{
T B Babel, P C Warnke, C B Ostertag
}

\begin{abstract}
Objective-The surgical treatment of intractable Tourette's syndrome is controversial. Experience with 17 consecutive patients treated between 1970 and 1998 is reviewed and the efficacy and safety of surgical treatment is assessed.

Methods-These patients were retrospectively reclassified into subtypes according to the protocol of the Tourette's Syndrome Study Group. One patient was excluded from the study. Ventriculography based stereotactic zona incerta (ZI) and ventrolateral/ lamella medialis thalamotomy (VL/LM) were performed on all patients. The preoperative, postoperative, and late tic severities were assessed by the tic severity rating scale. The median follow up of 11 patients $(65 \%)$ was 7 years (range 3.5-17 years) and six patients were lost to long term follow up.

Results-Median age was 23 years (range 11-40) at the time of surgery. Median duration of illness was 14 years (range 3-33). The mean preoperative motor and vocal tic severities were estimated to be 4.44 (SD 0.63) and 3.81 (SD 0.66), respectively. Unilateral ZI lesioning and VL/LM lesioning selected by asymmetry of symptoms provide an effective control of tic severity ( $p$ motor and vocal<0.001). In attenuation of contralateral symptoms, a second surgical intervention in the relevant side could reduce tic severity sufficiently (p motor $<0.01$; p vocal $<0.005$ ). Transient complications occurred in $68 \%$ of patients. Only one permanent complication was registered in six patients followed up after unilateral surgery. Two out of five patients followed up after bilateral surgery had disabling side effects of surgery.
\end{abstract}

Conclusions-ZI and VL/LM lesioning provide a significant long term reduction of tic severity in intractable Tourette's syndrome. Adequate selection of the side of first intervention might prevent the patient from increased risk of bilateral surgery.

(F Neurol Neurosurg Psychiatry 2001;70:666-671)

Keywords: Gilles de la Tourette's syndrome; infrathalamic and thalamic lesioning

pondence to:

Dr C B Ostertag

ostertag@nz.ukl.uni-freiburg.de

Received 31 May 2000 and in revised form

10 November 2000

Accepted 5 December 2000
The interpretation of Gilles de la Tourette's syndrome has changed radically since the first description of the disease in $1885 . .^{1-3}$ Recent studies suggest that Tourette's syndrome and chronic tic disorders seem to follow an autosomal dominant pattern of inheritance, with varying degrees of penetrance. Its expression is influenced by sex, with male preponderance. ${ }^{4-6}$ Tourette's syndrome is the most complex manifestation of a group of related tic disorders. The character of the tics is similar throughout the range, and it is one of the fastest growing diagnoses with a prevalence of from 2.9 to 5.2/10 000 population. ${ }^{78}$ Tic disorders usually develop in childhood and they will remit completely in $30 \%-40 \%$ of patients by late adolescence with an additional further improvement of $30 \%$ in later years. ${ }^{9}$ Drug therapy, driven by the most functionally disabling symptoms, consists of various therapeutic agents including haloperidol and pimozide. ${ }^{10}$ Despite the best psychopharmacological and behavioural therapeutic efforts, a few patients have residual debilitating symptoms, including self injurious behaviour. The management of this malignant manifestation of Tourette's syndrome remains controversial and a minority of patients are finally referred for surgery. ${ }^{11-14}$

Surgical results with intractable Tourette's syndrome are mostly anecdotal, without any firm statistical conclusions. ${ }^{15}$ We summarise our experience with 17 consecutive patients with intractable Tourette's syndrome treated by infrathalamic and/or thalamic lesioning. Our primary aim was to assess the feasibility and safety of this method by retrospective evaluation of the clinical and follow up data. In addition, we describe the potential characteristics of malignant Tourette's syndrome. Target selection and the validity of zona incerta (ZI) lesioning are discussed.

Patients and methods

Seventeen patients with medically intractable Tourette's syndrome were treated at the Stereotactic and Functional Neurosurgery Department of Freiburg (Germany) between 1970 and 1998. Their records were retrieved from the Freiburg stereotactic functional neurosurgery database (FSFND) and were reclassified according to the protocol of the Tourette's Syndrome Study Group. ${ }^{16}$ Only those patients who fulfilled the minimal criteria for Tourette's syndrome were included in the study.

We applied a simple five point scoring system (tic severity rating scale, TSRS) to quantify preoperative and postoperative tic severity. ${ }^{17}$ The rating process was based on the complexity and frequency of motor and vocal tic 
Table 1 Summary of clinical data and follow up time*

\begin{tabular}{llllllll}
\hline Patient No & Age $(y) /$ sex & Tics & Duration $(y)$ & Onset $(y)$ & History/age & Diagnosis & Follow up (y) \\
\hline 1 & 21/M & MT/VT & 12 & 9 & Head injury/15 y & TS & 17 \\
2 & 29/F & MT/VT & 16 & 12 & Tonsillitis/12 y & TS & \\
3 & 28/M & MT/VT & 14 & 4 & No data & TS & \\
4 & 21/F & MT/VT & 13 & 6 & No data & TS & 13 \\
5 & MT/F & MT/VT & 23 & 8 & No data & TS & 13 \\
6 & 34/F & MT/VT & 12 & 6 & No data & TS & \\
7 & 19/M & MT/VT & 14 & 5 & Pneumonia, epileptic fits/2 y & TS & 7 \\
8 & MT/FT & 33 & 7 & No data & TS & 8,5 \\
9 & 11/M & MT/VT & 7 & 4 & Pneumonia/4 months & TS & 9,5 \\
10 & 15/M & MT/VT & 3 & 12 & Epileptic fits/15 months & TS & 5,5 \\
11 & 20/M & MT/VT & 9 & 11 & Diphtheria/10 months & TS & 3,5 \\
12 & 23/M & MT/VT & 17 & 6 & No data & TS & 7 \\
13 & 21/M & MT/VT & 15 & 6 & Unspecified virus infection/5 y & TS & 4,5 \\
14 & 23/M & MT/VT & 19 & 4 & No & TS & 4,5 \\
15 & 26/M & MT/VT & 18 & 8 & No & STD & 7 \\
16 & 19/M & MT/VT & 13 & 6 & No & Unclear &
\end{tabular}

ॠThe only patient with non-idiopathic tic disorder (STD) was not included in the study. The aetiology of tics in this patient remained unclear; MT=motor tic; VT=vocal tic; TS=Tourette's syndrome.

phenomena. The assessment was carried out independently by two of us (TBB, PCW) not involved in the surgery. The different values from the rates were then combined and averaged. We compared preoperative, immediate, and late outcome TSRS scores by paired $t$ test. The same process was applied to preoperative and to late outcome scores in unilateral and bilateral surgical groups. A p value $<0.05$ was considered to be significant.

Five out of 16 patients ( $31 \%$ ) were lost for late outcome assessment. The median follow up of the remaining 11 patients was 7 years (range 3.5-17 years). The follow up period took place between 1990 and 1999. The neurological status, tic severity, and treatment satisfaction index (TSI) were registered in the FSFND.

INDICATION AND OPERATIVE TECHNIQUE

All patients referred to surgery were previously treated by multiple non-surgical methods (multidrug protocols, behavioural therapy, psychoanalysis, hypnosis etc) without any documented long term benefit. The side of the surgery was selected by the asymmetry of clinical manifestations of Tourette's syndrome. Second stage surgery was only indicated when the contralateral symptoms increased to a disabling level after the first intervention.

All patients were operated on under local anaesthesia with light sedation. The target coordinates were computed by ventriculography using the Riechert-Mundinger System (Fischer Co, Germany). The ventral border of the foramen of Monro (FM) and posterior comissure (PC) line was determined. ${ }^{18} \mathrm{ZI}$ was targeted at $12 \mathrm{~mm}$ behind the ventral border of FM, 3-5 mm below the FMPC line, and 8-9 $\mathrm{mm}$ lateral to the border of the third ventricle. The lamella medialis thalamus (LM) was selected at $7 \mathrm{~mm}$ posterior to the FM, $4 \mathrm{~mm}$ above the FMPC line and $6.5 \mathrm{~mm}$ lateral to the border of the third ventricle. The coordinates of the ventrolateral nuclei (VL) were computed as $10-13 \mathrm{~mm}$ behind the FM, between 0 and $1.5 \mathrm{~mm}$ from the FMPC line, and $9-10 \mathrm{~mm}$ lateral to the border of the third ventricle. Multiple radiofrequency lesions (3-4) up from the target site were performed using a string electrode (1 $\mathrm{mm}$ diameter, 5-7 $\mathrm{mm}$ exposed tip) and a thermocontrolled straight electrode (2 $\mathrm{mm}$ diameter $2 \mathrm{~mm}$ exposed tip) (Fischer Co, Germany). The lesioning indices were chosen as $80-90 \mathrm{~mA}, 25 \mathrm{~V}, 20-25$ seconds. Nine of 16 patients (56\%) were operated on bilaterally. ZI lesioning was performed in all but one patient (patient 7). ZI and VL/LM targets were selected in 11 patients (69\%).

\section{Results}

PATIENT CHARACTERISTICS

The relevant clinical data of patients and time of follow up are shown in table 1 . Median age of 16 patients ( 11 male and five female) at the time of surgery was 23 years (range 11-40). The median onset of the symptoms before surgery was computed as 6 years (range 4-12). The median duration of disease was 14 years (range 3-33 years). Neurological deficits and any abnormality disclosed by ventriculography, EEG, or CT were not registered. Brain MRI in patient 7 showed bilateral calcification of the basal ganglia. All but one patient did not meet the current diagnostic criteria for Tourette's syndrome. Attention deficit hyperactivity disorder and obsessive compulsive disorder were documented in patients 11,14 , and 15 .

\section{POSTOPERATIVE AND LATE TIC SEVERITY}

REDUCTION

The immediate tic severity reduction is shown in table 2. The late tic severity reduction in the follow up group of patients is presented in table 3. The significant tic severity reduction was found to be long lasting. Our results did not show any difference between motor and vocal tic severity control. All patients, except two in the follow up group, seemed to be satisfied with

Table 2 Immediate results in total group of patients

\begin{tabular}{lllll}
\hline & \multicolumn{2}{l}{ Score } & & \\
\cline { 2 - 4 } & Range & Preoperative & Postoperative & $p$ Value \\
\hline Motor tic score & $1-5$ & $4.4(0.63)$ & $1.2(0.4)$ & $<0.001$ \\
Vocal tic score & $1-5$ & $3.8(0.66)$ & $1.1(0.34)$ & $<0.001$
\end{tabular}

The immediate results showed significant reduction of tics by paired $t$ test.

Numbers in parentheses are SD. 
Table 3 Late results in follow up group of patients

\begin{tabular}{lllll}
\hline & \multicolumn{2}{c}{ Score } & & \\
\cline { 2 - 4 } & Range & Preoperative & Late & p Value \\
\hline Motor tic score & $1-5$ & $4.6(0.5)$ & $2.3(0.9)$ & $<0.001$ \\
Vocal tic score & $1-5$ & $3.7(0.65)$ & $1.6(0.67)$ & $<0.001$
\end{tabular}

The late tic reduction was significant by paired $t$ test. Numbers in parentheses are SD.

Table 4 Late results after bilateral interventions

\begin{tabular}{lllll}
\hline & \multicolumn{2}{l}{ Score } & & \\
\cline { 2 - 4 } & Range & Preoperative & Late & p Value \\
\hline Motor tic score & $2-5$ & $4.4(0.55)$ & $2.8(0.45)$ & $<0.01$ \\
Vocal tic score & $1-5$ & $3.6(0.89)$ & $1.6(0.55)$ & $<0.005$ \\
\hline
\end{tabular}

The late results showed that the most significant improvement was the reduction of vocal tics.

Numbers in parentheses are SD.

Table 5 Late results after unilateral infrathalamic and thalamic lesioning

\begin{tabular}{lllll}
\hline & \multicolumn{2}{c}{ Score } & & \\
\cline { 2 - 4 } & Range & Preoperative & Late & p Value \\
\hline Motor tic score & $1-5$ & $4.6(0.5)$ & $2.3(0.9)$ & $<0.001$ \\
Vocal tic Score & $1-4$ & $3.7(0.65)$ & $1.7(0.79)$ & $<0.001$ \\
\hline
\end{tabular}

The late results in this group showed that the most significant improvement was the reduction of vocal tics. The alleviation of motor tics was less predictable but was significant.

Numbers in parentheses are SD.

treatment. Interestingly, the sensory tic phenomena were also improved in patient 11 . Almost all patients remained on multidrug therapy. The late reduction in tic severity in the group of patients operated on bilaterally or unilaterally is shown in tables 4 and 5. The alleviation of vocal tics was more significant than that of motor tics. The reduction in motor tic severity in the bilateral surgical group was less significant than in the unilateral group. Unilateral ZI and VL/LM lesioning selected by asymmetry of symptoms provided an effective control of tic severity. In cases of contralateral symptom attenuation, surgical intervention on the relevant side could sufficiently reduce tic severity.

Table 6 Summary of the target sites and complications of bilateral and unilateral interventions

\begin{tabular}{|c|c|c|c|c|c|}
\hline Patient No & $Z I$ & $V L$ & $L M$ & Postoperative complications & Late complications \\
\hline 2 & $\mathrm{R} / \mathrm{L}$ & $\mathrm{R}$ & & not mentioned & \\
\hline 3 & $\mathrm{R} / \mathrm{L}$ & $\mathrm{R} / \mathrm{L}$ & & not mentioned & \\
\hline 4 & $\mathrm{~L}$ & $\mathrm{~L}$ & $\mathrm{R} / \mathrm{L}$ & Dystonia & \\
\hline 8 & $\mathrm{R} / \mathrm{L}$ & & & Not mentioned & \\
\hline 9 & $\mathrm{R} / \mathrm{L}$ & & & Dystonia $\mathrm{R}$ & Improved \\
\hline 10 & $\mathrm{R} / \mathrm{L}$ & & & Dysarthria, dystonia & Improved \\
\hline 11 & $\mathrm{R} / \mathrm{L}$ & & & Dysarthria, dystonia, CS & Unchanged \\
\hline 12 & $\mathrm{R} / \mathrm{L}$ & $\mathrm{R} / \mathrm{L}$ & & Not mentioned & No deficit \\
\hline $13^{\star}$ & $\mathrm{R} / \mathrm{L}$ & $\mathrm{R} / \mathrm{L}$ & & Dysarthria, hemiballisms, CS & Unchanged \\
\hline 1 & $\mathrm{R}$ & & $\mathrm{R}$ & Mild hemiparesis L & Improved \\
\hline 5 & $\mathrm{~L}$ & $\mathrm{~L}$ & $\mathrm{~L}$ & Dystonia & Improved \\
\hline 6 & $\mathrm{~L}$ & $\mathrm{~L}$ & $\mathrm{~L}$ & Not mentioned & No deficit \\
\hline 7 & & $\mathrm{R}$ & $\mathrm{R}$ & Dysartria, mild hemiparesis L & \\
\hline 14 & $\mathrm{R}$ & $\mathrm{R}$ & & Hemiparesis R, CS & Unchanged \\
\hline 15 & $\mathrm{~L}$ & $\mathrm{~L}$ & & CS & No deficit \\
\hline 16 & $\overline{\mathrm{R}}$ & $\overline{\mathrm{R}}$ & $\mathrm{R}$ & Dystonia, CS & No deficit \\
\hline
\end{tabular}

The postoperative complications in bilateral cases are summarised after second stage intervention. $\mathrm{ZI}=$ Zona incerta; $\mathrm{VL}=$ nucleus ventrolateralis; $\mathrm{LM}=$ nuclei lamina medullaris thalami; $M T=$ motor tics; VT=vocal tics; $\mathrm{R}=$ right; $\mathrm{L}=$ left.

${ }^{\star}$ During the second stage infrathalamic lesioning severe hemiballism was detected as a consequence of subthalamic nucleus coagulation. Further lesioning was carried out in an attempt to alleviate the symptom.
POSTOPERATIVE AND LATE COMPLICATIONS

There was no operative mortality. Cerebellar signs, dystonia, and dysarthria were detected in 11 of the total group $(69 \%)$ and in nine of 11 patients in the follow up group (81\%). Postoperative morbidity in six of nine patients $(66 \%)$ proved to be transient (table 6). Three patients in the follow up group (27\%) had disabling side effects of surgery. In patient 13, an intraoperative complication of contralateral ZI lesioning resulted in disabling hemiballism. In patient 14 , after a very transient improvement from left $\mathrm{ZI}$ and $\mathrm{VL}$ lesioning, the patient refused the contralateral operation.

\section{Discussion}

The pathology of Tourette's syndrome has not been clearly defined. A decrease in volume of the corpus striatum due to underdevelopment, with additional gliosis in the ventromedial nuclei of the thalamus, has been described. ${ }^{19}{ }^{20}$ Brain MRI sometimes shows a significant reduction in left striatal-pallidal volumes, with deviations of lateral ventricular volume and asymmetry in patients with Tourette's syndrome compared with normal controls. ${ }^{21} 22$ These findings led to speculation on brain lateralisation in the disease and suggested a role for sex hormones as relevant developmental factors. ${ }^{23}$ In the group that underwent unilateral surgery we were not able to identify any obvious laterality. Four male patients had surgery on the right, but only one of the three patients with left side surgery was male. (table 6 ). Adequate selection of the side of first intervention might prevent the patient from increased risk of further surgery. ${ }^{24}$ Basal ganglia $M R$ volumetry and SPECT/PET studies might improve the side selection for surgery.

DIAGNOSIS OF TIC DISORDERS

Tourette's syndrome was initially diagnosed by the clinical manifestations of the illness, resulting in much confusion in clinical practice. ${ }^{3}$ Tic disorders have recently been redefined by various complex diagnostic criteria and the time course of tic phenomena. ${ }^{16}$ Tourette's syndrome is commonly accompanied by various behavioural disturbances - for example, obsessive compulsive disorder and attention deficit hyperactivity disorder. ${ }^{925}$ Sensory tics, reported to be a common and distinctive feature of Tourette's syndrome, are defined as the subjectively experienced component of neural dysfunction below the threshold for motor and vocal tic production. ${ }^{26}$ Sensory tics and overlapping obsessive compulsive disorder phenomena are not consistently delineated. Tics may also accompany various other neurological conditions and are than classified as secondary tic disorders. This condition is usually seen in conjunction with other movement disorders.

There are various possible underlying conditions that may result in the symptoms described as tic disorders. Recent research has implicated group A $\beta$ haemolytic streptococcus infection (GABHS) as a possible environmental trigger in families genetically susceptible to various movement disorders, including 


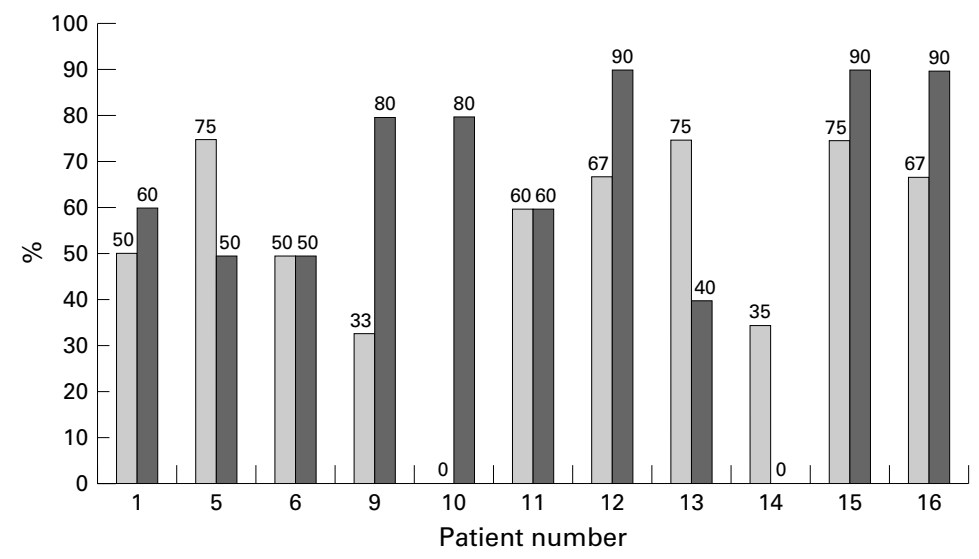

Figure 1 Comparison of the rate of reduction in vocal tic severity with the treatment satisfaction index (TSI) disclosed controversies of outcome assessment. TSI is a simple visual scale from absent (0) to absolute satisfaction (100\%) estimating satisfaction with the result of surgical treatment. Light columns =tic severity reduction rate; dark columns $=$ treatment satisfaction index.

Tourette's syndrome. ${ }^{27}{ }^{28}$ However, these findings might explain only a part of its aetiology. The artificial separation of Tourette's syndrome, involuntary movement disorders, and compulsive phenomena are not consistently supported by clinical evidence. ${ }^{13} 2930$ Interestingly, our patients' records did not disclose any direct evidence of hereditary transmission, except for patient 14; mild head jerking by the patient's father was registered. Possible GABHS infection might have played a part in admission to hospital for pneumonia in patients 7 and 10 and tonsillectomy in patient 2 (table 1). In this patient, tics appeared in the year when a severe tonsillitis was finally operated on. The sex distribution in our case series and the time onset of symptoms was in accordance with the literature. ${ }^{925}$ On the other hand the overlapping of obsessive compulsive disorder and attention deficit hyperactivity disorder were documented in only three patients. ${ }^{15}$

Malignant Tourette's syndrome may represent a special subgroup of the tic range. ${ }^{912} 14$ The controversies about management of this subgroup make a strong case for investigation separately from the less florid manifestation of the syndrome. Typical malignant Tourette's syndrome is characterised as a medically intractable condition with a persistently high

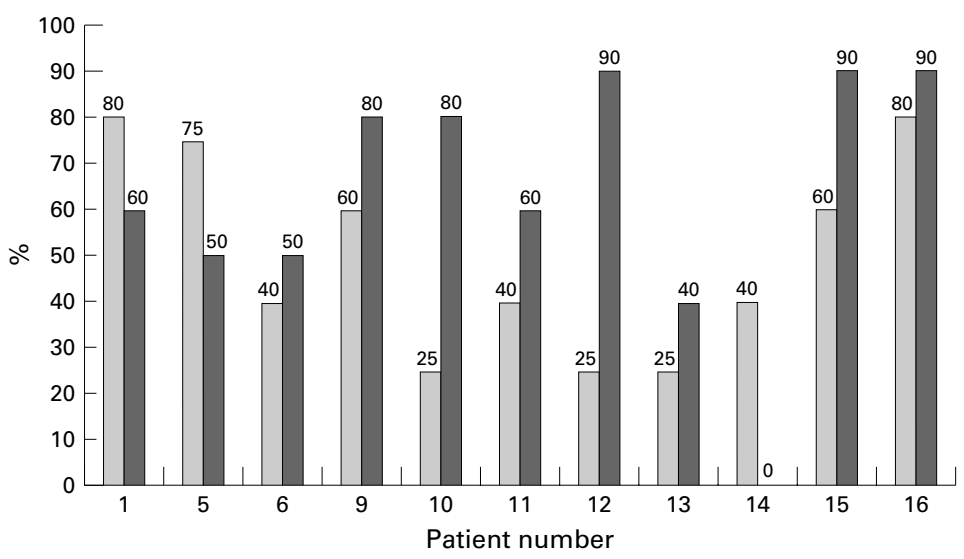

Figure 2 Comparison of the rate of motor tic severity reduction with the treatment satisfaction index (TSI). Further explanation as figure 1. score on tic severity rating scales, resulting in self injurious behaviour or severe impairment of social function. Disappearance of waxing and waning phenomena in the dynamics of Tourette's syndrome was documented in all patients. Management of these young patients with malignant Tourette's syndrome ( median age at surgery 23 years) could be very difficult because of the lifelong nature of the illness. The retrospective nature of the study does not allow us to give clear cut selection criteria for surgery. However, based on our findings, we propose to include the permanence of a high score in TSRS with lack of waxing and waning phenomena with a documented medically intractable condition.

\section{OUTCOME ANALYSIS}

Tics are probably the most difficult of all movement disorders to rate with validity and reliability. ${ }^{17}{ }^{31}{ }^{32}$ They are remarkably variable in their clinical range and most patients have some temporary volitional control over their movements. Permanent disabling tic symptoms were documented in all our patients. The retrospective approach of the study allowed the use of a simple tic severity assessment. In this context our results indicate that the reduction in tic severity after ZI and VL/LM lesioning remains long lasting. The motor tic reduction seemed to be less predictable but significant. We were not able to detect a best responder subgroup within our patients. In half of our patients contralateral intervention was indicated to alleviate the attenuated contralateral symptoms. The bilateral interventions could provide effective long term tic reduction but there is a higher risk for permanent morbidity. Lesioning of the ZI and VLT is clearly a procedure with higher risk, but more than 50\% immediate morbidity is transient. ${ }^{33} 34$

Despite the nowadays unacceptable high morbidity risk almost all patients seemed to be satisfied with their treatment. The further evaluation of follow up data suggest that the fluctuation of symptoms of Tourette's syndrome remained below the disabling level, allowing the patients to function independently after surgical intervention and to respond again to non-surgical treatments. However, our long term results seem to challenge using reduction of symptoms as the only methodology for outcome assessment. Even with very simple dual outcome assessment, we were not able to detect an association between reduction in tic severity and patient satisfaction (figs 1 and 2). Our findings suggest a careful interpretation of long term outcome data that should in the future include the quality of life rating scales.

TARGET SELECTION

The relevant neuroanatomical model for Tourette's syndrome surgery has been intensively reviewed by Rauch et al. ${ }^{15}$ Only the recent contributions are summarised here. Brain PET studies have provided data suggesting that the striatolimbic-premotor area is uncoupled in Tourette's syndrome compared with control subjects. However, the metabolic rate of the 
striatal-sensorimotor region was positively correlated with Tourette's syndrome..$^{35}$ The ventral striatum, corpus callosum, thalamus, and midbrain were reported as potentially important neural substrates of Tourette's syndrome..$^{25} 3637$ All structures have neuroanatomical and functional connections to the corticostriatalthalamocortical circuitry. The basal ganglia contain multiple output channels and their dysfunction might result in motor or cognitive dysfunction. ${ }^{38-41}$ In this context, involuntary movements are identified as a consequence of disruption of the normal control of the basal ganglia. The abnormal pattern of neuronal discharge in the motor circuit could lead to involuntary activation of fragments of movement. ${ }^{42}$ This would be consistent with the recent findings that primary motor and premotor areas control sets of synergistic muscles rather than a single muscle or just one specific action. ${ }^{43}$ Tourette's syndrome might be interpreted as an overriding abnormal neural activity of the motor circuit involving multiple outputs from the basal ganglia.

The ZI and VL/LM lesioning has been earlier reported as an alternative approach for controlling involuntary movements. ${ }^{3044} 45$ The role of intralaminar nuclei (LM) in pathogenesis and in surgery for Tourette's syndrome has been stressed previously. ${ }^{24}{ }^{46}{ }^{47} \mathrm{VL} / \mathrm{LM}$ surgery alone was performed in one out of 16 patients (patient 7 , table 6). Consequently we were not able to compare thalamic targets after infrathalamic lesioning. Additionally, ZI lesioning, as a separate target from the subthalamotomy, has to be delineated. The infrathalamic region, defined as an area between the thalamus and subthalamic nucleus (STN), consists of ZI and the main output and input pathways within pallidal-subthalamothalamic and thalamiccerebellar circuits. The volume of the $\mathrm{ZI}$ is highly variable. ${ }^{48}$ The primary effect of $\mathrm{ZI}$ lesioning could result in alteration of basal ganglia output rather then in ZI lesion itself (fig 3)..$^{1544649}$ This view might be supported by different immediate side effects of pallidotomy, subthalamotomy, and ZI lesioning (table 6). ${ }^{34445051}$ In our series the reported intraoperative complication of hemiballism was due to STN coagulation. Future lesion position studies might clear the validity of basal ganglia output lesioning as named as infrathalamotomy against subthalamotomy.

Four approaches are currently available for neurosurgical treatment of intractable Tourette's syndrome: psychiatric surgery (limbic leucotomy, cingulotomy), ZI and VL/LM lesioning, and a novel combination of cingulotomy and infrathalamic lesioning. ${ }^{12} 334452$ Recently reported high frequency stimulation (deep brain stimulation) of the right thalamic intralaminar nuclei seemed to reduce the risk of permanent morbidity and inadequate side selection. ${ }^{24}$ None of the methods has been found to be superior by valid statistical methodology. ${ }^{15}$ The $\mathrm{ZI}$ and VL/LM targets could be chosen for alleviating the involuntary movement component of Tourette's syndrome symptomatology. The psychiatric surgery might improve effectively the overlapping obsessive

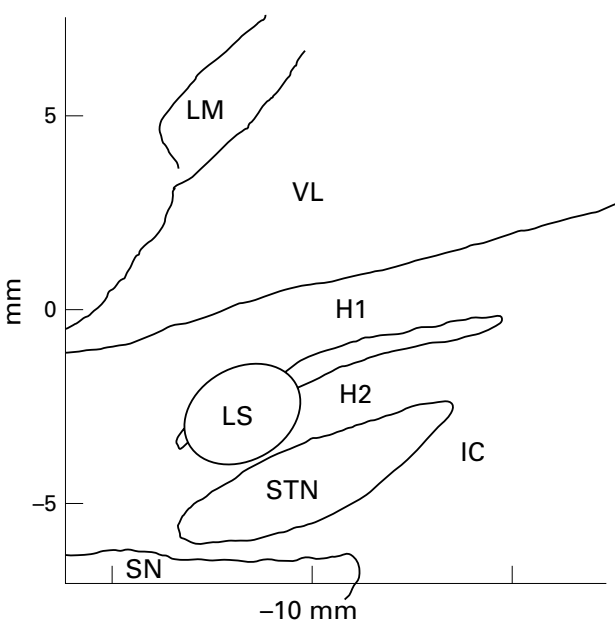

Figure 3 Depiction of the zona incerta exact lesion site in the sagittal plane at the coordinates $12 \mathrm{~mm}$ behind the ventral border of the foramen of Monro, 3-5 $\mathrm{mm}$ under the foramen of Monro and posterior comissure line and $9 \mathrm{~mm}$ lateral to the border of the third ventricle. Infrathalamic lesioning might primarily affect the basal ganglia output pathways. $V L=$ nucleus ventrolateralis thalami;

$L M=$ lamina medullaris thalamus; $S T N=$ subthalamic nucleus; $S N=$ substantia nigra; $I C=$ internal capsule; $H 1$ and $\mathrm{H}_{2}=$ fields of Forel.

compulsive behaviour phenomena. ${ }^{12}{ }^{52-55}$ Target selection might be based on the most functional disabling symptoms of malignant Tourette's syndrome.

\section{Conclusions}

Medically intractable Tourette's syndrome, here defined as malignant Tourette's syndrome, shows progression in tic symptomatology and conversion of waxing and waning phenomena. Our results indicate that unilateral ZI and VL/LM lesioning selected by asymmetry of symptoms provided an effective control of tic severity. The long term motor tic reduction seemed to be less predictable but significant. More than $50 \%$ of immediate morbidity after $\mathrm{ZI}$ and VL/LM lesioning was transient. In cases of attenuation of contralateral symptoms, surgical intervention on the relevant side could efficiently reduce tic severity but with higher risk of permanent morbidity. The exact target site of infrathalamic lesion needs to be delineated. An adequate selection of the side of first intervention might prevent the patient from increased risk of further surgery. Neuroimaging outcome studies may clarify the exact role for neuroimaging in target and side selection. Deep brain stimulation techniques might lower the risk of inadequate side selection and permanent morbidity. A prospective randomised study including the quality of life domain is needed to validate the role of surgery in the management of malignant Tourette's syndrome.

The present work was supported by DAAD grant No A/99/06567. We thank Dr Elias Milios for his collaboration.

1 Gilles de la Tourette. Etude sur une affection nerveuse caracterisee par de l'incoordination motrice accompagnee d'echolalie et de copralalie. Arch Neurol (Paris) 1885;9:1942,158-200.

2 Itard JMG. Memoire sur quelques fonctions involontaires des appareils de la locomotion, de la prehension, et de la
voix. Archives Générales de Médicine (Paris) 1825;8:385voix. 

3 Kushner HI. A cursing brain. Boston: Harvard University

4 Marsden CD, Obeso JA. The function of the basal ganglia and the paradox of stereotactic surgery in Parkinson's disease. Brain 1994;117:877-97.

5 Pauls DL, Leckman JF. The inheritance of Gilles de la Tourette's syndrome and associated behaviors: evidence for autosomal dominant transmission. $N$ Engl F Med 1986;315 993-7.

6 Pauls DL, Pakstis AJ, Kurlan R, et al. Segregation and linkage analysis of Tourette' syndrome and related disorders. $\mathcal{F}$ Am Acad Child Adolesc Psychiatry 1990;29:195-203.

7 Eapen V, Robertson MM, Zeitlin H, et al. Gilles de la Tourette's syndrome in special education schools: a United Kingdom Study. F Neurol 1997;244:378-82.

8 Tanner CM, Goldman SM. Epidemiology of Tourette syndrome. Neurol Clin 1997;15:395-402.

9 Bruun RD, Budman CL. The natural history of Tourette syndrome. Adv Neurol 1992;58:1-6.

10 Kurlan R, Trinidad KS. Treatment of tics. In: Kurlan R, ed. Treatment of movement disorders. Baltimore: JB Lippincott, 1995:365-406.

11 Baer L. Behavior therapy for obsessive-compulsive disorder . Implications for Tourette syndrome. Adv Neurol 1992;58:333-40.

12 Kurlan R, Kersun J, Ballantine Th Jr, et al. Neurosurgical treatment of severe obsessive-compulsive disorder associated with Tourette's syndrome. Mov Disord 1990;2:152-5.

13 Kurlan R: Hypethesis II. Tourette's syndrome is a part of a clinical spectrum that includes normal brain development. Arch Neurol 1994;51:1145-50.

14 Robertson MM. Self-injurios behavior and Tourette syndrome. Adv Neurol 1992;58:105-14.

15 Rauch SL, Baer L, Cosgrove GR, et al. Neurosurgical treatment of Tourette's syndrome: a critical review. Compr Psychiatry 1995;36:141-56.

16 The Tourette Syndrome Classification Study Group. Definitions and classification of tic disorders. Arch Neurol 1993;50:1013-16.

17 Goetz CG, Tanner CM, Wilson RS, et al. A rating scale for Gilles de la Tourette's syndrome: description, reliability, Gilles de la Tourette's syndrome: descripti

18 Hassler R, Riechert T. Indikationen und Lokalisationsmethode der gezielten Hirnoperationen. Nervenarzt 1954;25 $441-7$

19 Balthasar K. Ueber das anatomische Substrat der generalisierten Tic-Krankheit (maladie des tics, Gilles de la Tourette). Archiv für Psychiatrie und Zeitschrift Neurologie 1957; 195:531-49.

20 Clauss JL, Balthasar K. Zur Kenntnis der generalisierten Tic-Krankheit (maladie des tics, Gilles de la Tourette'sche Krankheit). Archiv für Psychiatrie und Zeitschrift Neurologie 1954;191:398-418.

21 Peterson B, Riddle MA, Cohen DJ, et al. Reduced basal ganglia volumes in Tourette's syndrome using threedimensional reconstruction techniques from magnetic resonance images. Neurol 1993;43:941-9.

22 Singer HS, Reiss AL, Brown JE, et al. Volumetric MRI changes in basal ganglia of children with Tourette's changes in basal ganglia of child

23 Witelson SF. Clinical neurology as data for basic neuroscience: Tourette's syndrome and the human motor neuroscience: Tourette's syndrom

24 Vanderwalle V, van der Linden Chr, Groenewegen HJ, et al. Stereotactic treatment of Gilles de la Tourette syndrome by high frequency stimulation of thalamus. Lancet 1999;353 724

25 Jankovic J. Tics and Tourette's syndrome. In: Tolosa E, Koller WC, Gershanik OS, eds. Differential diagnosis and treatment of movement disorders. Oxford: ButterworthHeinemann, 1998:101-10.

26 Chee KY, Sachdev P. A controlled study of sensory tics in Gilles de 1a Tourette syndrome and obsessive-compulsive disorder using a structured interview. $\mathcal{F}$ Neurol Neurosurg Psychiatry 1997;62:188-92.

27 Allen AJ, Leonard HL, Swedo SE. Case study: a new infection-triggered, autoimmune subtype of pediatric OCD infection-triggered, autoimmune subtype of pediatric OCD try 1995;34:307-11.

28 Swedo SE, Leonard HL, Mittleman BB, et al. Identification of children with pediatric autoimmune neuropsychiatric disorders associated with streptococcal infections by a marker associated with rheumatic fever. Am f Psychiatry 1997;154:110-12.
29 Jankovic J, Ashizawa T. Tourettism associated with Huntington's disease. Mov Disord 1995;10:103-5.

30 Nemeth AH, Mills KR, Elston JS, et al. Do the same genes predispose to Gilles de la Tourette syndrome and dystonia? Report of a new family and review of the literature. Mov Disord 1999;14:826-31.

31 Goetz CG, Stebbins GT, Thelen JA. Talixepole and adult Gilles de la Tourette's syndrome: double-blind, placebocontrolled clinical trial. Mov Disord 1994;3:315-17.

32 Leckman JF, Riddle MA, Hardin MT. The Yale global tic severity scale: initial testing of a clinician rated scale of severity. I Am Acad Child Adolesc Psychiatry 1989;28:56673.

33 Leckman JF, de Lotbiniére AJ, Marek K, et al. Severe disturbances in speech, swallowing, and gait following stereotacic infrathalamic lesions in Gilles de la Tourette's syndrome. Neurology 1993;43:890-94.

34 Lee MS, Marsden CD. Movement disorders following lesions of the thalamus or subthalamic region. Mov Disord 1994;9:493-507.

35 Stoetter B, Braun AR, Randolph Ch, et al. Functional neuroanatomy of Tourette syndrome. Limbic-motor interactions studied with FDG PET. Adv Neurol 1992;58:213-26.

36 Haber SN, Wolfer D. Basal ganglia peptidergic staining in
Tourette syndrome. Adv Neurol 1992;58:145-50. Peterson BS, Bronen RA, Duncan CC. Three cases of symptom change in Tourette's syndrome and obsessivecompulsive disorder associated with paediatric cerebral malignancies. F Neurol Neurosurg Psychiatry 1996;61:497505.

38 Alexander GE. Basal ganglia-thalamocortical circuits: their role in control of movements. F Clin Neurophysiol 1994;11: $420-31$.

39 Hoover JE, Strick PL. Multiple output channels in the basal ganglia. Science 1993;259:819-21.

40 Middleton FA, Stick PL. New concepts about the organization of basal ganglia output. Adv Neurol 1997;74:57-68.

41 Mundinger F, Riechert T, Disselhoff J. Long term results of stereotactic operations on extrapyramidal hyperkinesia (excluding Parkinsonism). Confina Neurologica 1970;32: $71-8$.

42 Obeso JA, Rodriguez MC, DeLong MR. Basal ganglia pathophysiology. A critical review. Adv Neurol 1997;74:318 .

43 Baraniga M. Remapping of motor cortex. Sience 1996;268: 1696-8.

44 Hassler R, Riechert T, Mundinger F, et al. Physiological observations in stereotaxic operations in exptrapyramidal motor disturbances. Brain 1960;83:337-50.

45 Hassler R, Mundinger F, Riechert T. Stereotaxis in Parkinson syndrome. Wien: Springer Verlag, 1979:231-2.

46 Hassler R, Dieckmann G. Traitement stereotaxique des tics et cris inarticules ou coprolalique consideres comme phenomene d'obsession motrice au cours de la maladil

47 Leckman JF, Pauls DL, Peterson BS, et al. Pathogenesis of Tourette syndrome. Clues from the clinical phenotype and natural history. Adv Neurol 1992;58:15-24.

48 Andrew J, Watkins ES. A stereotactic atlas of the human thalamus and adjecent structures. A variability study. Baltimore: Williams and Wilkins, 1969:107-12.

49 Middleton FA, Stick PL. Anatomical evidence for cerebellar and basal ganglia involvement in higher congnitive function. Science 1994;266:458-61.

50 Guridi J, Obeso JA. The role of the subthalamic nucleus in the origin of hemiballism and parkinsonism: new surgical perspectives. Adv Neurol 1997;74:235-47.

51 Kelly PJ. Pallidotomy in Parkinsons' disease. Neurosurg 1999;36:1154-7.

52 Robertson MM, Doran M, Trimble M, et al. The treatment of Gilles de la Tourette syndrome by limbic leucotomy $f$ Neurol Neurosurg Psychiatry 1990;53:691-4.

53 Cosgrove R, Ballantine HTh. Cingulotomy in psychosurgery. In: Gildenberg PhL, Tasker RR eds. Textbook of stereotactic and functional neurosurgery. New York: McGraw-Hill, 1998:1965-70.

54 Kulisevsky J, Berthier ML, Avila A. Longitudinal evaluation of prefrontal leucotomy in Tourette's syndrome. Mov Disord 1995;10:345-8.

55 Spangler WJ, Cosgrove GR, Ballantine $\mathrm{ThH} \mathrm{Jr}$, et al. Magentic resonance image-guided stereotactic cingulotomy for intractable psychiatric disease. Neurosurgery 1996;38:1071-8. 